\title{
Optimization of Replenishment Strategy based on Collaborative Planning Forecasting and Replenishment
}

\author{
Hui Pang ${ }^{a}$ and Shaohua Dong ${ }^{b,}{ }^{*}$ \\ School of Mechanical Engineering, University of Science and Technology Beijing, Beijing 100083, \\ P.R. China. \\ a moon2759@163.com, *,b dsh_dle@me.ustb.edu.cn
}

\begin{abstract}
Keywords: CPFR-based replenishment model, Coordination replenishment model, inventory management problem.
\end{abstract}

\begin{abstract}
Based on the mathematical programming method, this paper constructs the retail CPFRbased replenishment model, analyzes the determination of replenishment strategy, and tests the model. Then, through the analysis of the sensitivity of relevant parameters, the impacts of demand and product satisfaction rate on supply chain inventory cost and order quantity under the cooperative replenishment mode are studied. The research results show that the coordinative replenishment model can save the inventory management cost of the supply chain, strengthen the competitiveness and collaboration of the whole supply chain, and improve the customer satisfaction. The conclusion of the research is of practical significance and can provide reference for retail enterprises to implement the DPFR-based collaborative replenishment and solve the related inventory management problem to a certain extent.
\end{abstract}

\section{Introduction}

In the current inventory management, collaborative planning, forecasting, and replenishment (CPFR) are more advanced approaches. CPFR requires the enterprises at the supply chain to coordinate for the common interests of the supply chain, accomplishing the collaboration from the early plans to forecast, replenishment, and delivery. Compared to other inventory management theories, the CPFR theory has its own characteristics, including a wide range of collaborative thinking, a comprehensive inventory management plan, accurate demand forecast and the optimal replenishment strategy [1].

Collaborative replenishment is one stage in CPFR. The collaborative replenishment model is also based on the CPFR theory. Its implementation is on the basis of collaborative planning and forecasting [2]. In terms of the impacts of collaborative supply chain on the overall benefits of supply chain, Susan Li, Zhimin Huang and Allan Ashley studied the game between suppliers and manufacturers in a supply chain. By comparing the overall returns of the supply chain when there is collaboration or no collaboration, they concluded that collaboration can create higher supply chain return [3]. Barratt and Oliveira explored the possible problems during the CPFR implementation process and concluded that supply chain members should address these possible problems by continuously modifying the supply chain contract [4]. Steermainn explored how the retailer Seaus and its supplier Michelin deal with the coexistence of high inventory and out-of-stock in the transaction process through CPFR [5]. LinRong-Ho described the effects of CPFR on the purchase of the hospital and analyzed the changes of interests after implementing the CPFR through a model. CPFR can help the hospital reduce inefficient procurement and retain valuable medical resources [6].

CPFR has changed the company's inventory strategy, replenishment mode and forecasting methods, which has resulted in reducing inventory, increasing sales, and enhancing the relationship with trading partners [7], thus bringing great benefits to the entire value chain. 


\section{Model Parameter Description}

This paper assumes that the supply chain is a simple two-level supply chain with only one retailer and one supplier. The retailer takes the $(\mathrm{R}, \mathrm{Q})$ ordering strategy. If there is lead time for an order, the lead time does not change as well.

Before the implementation of collaborative replenishment, the retailer is at an advantage position in the supply chain. Its decision is decisive in the decision-making process. It determines the quantity of order from the supplier according to the principle of minimum inventory. After implementing the CPFR, the retailer and the supplier achieve the collaboration and make the decision of replenishment by following the principle of supply chain cost minimization.

Customers demand for the goods is affected by a variety of reasons, so the market demand is random. For the convenience of research, assuming that the random demand meets the normal distribution.

The unit cost of product does not change with the changes of inventory management decision. Therefore, the cost of product is not taken into account when calculating the cost of the inventory.

Table 1. Model Parameter Description

\begin{tabular}{|c|c|}
\hline Parameter & Description \\
\hline$D$ & Total annual demand, subject to the normal distribution $N\left(\mu, \sigma^{2}\right)$ \\
\hline$c_{S}$ & The cost of each order processing of the supplier, unit: Yuan/Order \\
\hline$c_{r}$ & The cost of every ordering of the retailer, unit: Yuan/Order \\
\hline$h_{S}$ & The storage fee of unit product of the supplier, unit: Yuan/Year/Piece \\
\hline$h_{r}$ & The storage fee of unit product of the retailer, unit: Yuan/Year/Piece \\
\hline$Q$ & Order quantity ( $Q^{*}$ is the optimal order quantity) \\
\hline$L$ & The lead time of an order \\
\hline$D_{L}$ & The demand at the lead time \\
\hline$\sigma_{L}$ & The standard deviation of demand at the lead time \\
\hline$R$ & Order point \\
\hline$S S$ & Safety stock \\
\hline$\lambda$ & Pafety coefficient (the number of standard deviations at established service levels) \\
\hline$\alpha$ & The number of outages per order cycle \\
\hline$U$ & The annual supply chain inventory management cost \\
\hline$T C$ & The supplier's annual inventory management cost \\
\hline$T C_{S}$ & The retailer's annual inventory management cost \\
\hline$T C_{r}$ &
\end{tabular}

Assume the mean of lead time demand $D_{L}$ is in normal distribution $\mu_{L}$ and the standard deviation is $\sigma_{L}$. Where $\mu_{L}$ and $\sigma_{L}$ can be obtained by the following formula:

$$
\begin{aligned}
& \mu_{L}=\mu L / 365 \\
& \sigma_{L}=\sigma \sqrt{\mathrm{L} / 365}
\end{aligned}
$$

The safety stock can be expressed as $\alpha \sigma_{\mathrm{L}}$, therefore the order point can be represented by the following formula:

$$
R=\mu_{L}+\alpha \sigma_{L}
$$

Product satisfaction rate refers to the probability that the product inventory meets the customers' needs. For retailers, satisfaction rate is more important than the level of customer service, which allows retailers to assess the sales demand. Besides, the improved satisfaction rate can lead to higher service level. The annual shortage can be expressed by $D$ and $\lambda$ as $D(1-\lambda)$. The quantity of each order is $Q$, then the number of orders per year is $D / Q$. For each order, the expected shortage is $E(U)$, then the annual shortage is $E(U) \times D / Q . E(U)$ Can be expressed as $E(\alpha) \sigma_{L}$. Therefore, the annual shortage can also be expressed as $E(\alpha) \sigma_{L} \times D / Q$. Then, from the above analysis we can see that $D(1-\lambda)=E(\alpha) \sigma_{L} \times D / Q$, namely:

$$
(1-\lambda) Q=E(\alpha) \sigma_{L}
$$


In order to get the order quantity $Q, E(\alpha)$ must be obtained first. $E(\alpha)$ Can be expressed by the probability $\alpha$ distribution function $F(\alpha)$ and the probability density function $f(\alpha)$. Introduce $E(U)$ and get:

$$
E(U)=f(\alpha) \sigma_{L}-\alpha[1-F(\alpha)] \sigma_{L}
$$

The formulas for $Q$ and $\alpha$ are given by the formula (4) and the formula (5) as follows.

$$
(1-\lambda) Q=f(\alpha) \sigma_{L}-\alpha[1-F(\alpha)] \sigma_{L}
$$

From the formula (6) we can see that the relationship between $Q$ and $\alpha$ is more complex.

\section{Establishment of the Model}

The idea of establishing a model is to obtain the quantity of order when the inventory cost is the minimum, by establishing an inventory-cost relationship formula. According to the assumptions of the model, before the implementation of CPFR, the retailer has a dominant position. The supplier can only replenish with the retailer's request. In the process of solving the model, the aim is to achieve the minimum stock of the retailer, thus determining the order quantity. After the implementation of CPFR, the sufficient information exchange and sharing between the retailer and the supplier has been established. On the basis of collaborative planning and forecasting, the replenishment strategy will be created with the purpose of minimizing the total stock cost of supply chain, thus determining the optimal order quantity.

(1) Before the implementation of collaborative replenishment

The retailer's annual stock management fee includes the cost of the order and the cost of storage.

The number of orders per year: $\mu / Q$

The average of annual stock: $Q / 2+\alpha \sigma_{L}$

Ordering cost: $c_{r} \mu / Q$

Storage cost: $h_{r} \times\left(Q / 2+\alpha \sigma_{L}\right)$

The retailer's annual stock management fee:

$$
T C_{r}=c_{r} \mu / Q+h_{r} \times\left(Q / 2+\alpha \sigma_{L}\right)
$$

The supplier's annual stock fee includes the order processing cost and the storage cost.

The order processing cost: $c_{s} \mu / Q$

The storage cost: $h_{s} Q / 2$

The supplier's annual stock management cost:

$$
T C_{s}=c_{s} \mu / Q+h_{s} \mathrm{Q} / 2
$$

According to the formula (7) and (8), the annual stock management cost of the supply chain is:

$$
\begin{gathered}
T C=T C_{r}+T C_{s}=c_{r} \mu / Q+h_{r}\left(Q / 2+\alpha \sigma_{L}\right)+c_{s} \mu / Q+h_{s} Q / 2 \\
=\left(c_{r}+c_{s}\right) \mu / Q+\left(h_{\mathrm{r}}+h_{\mathrm{s}}\right) \mathrm{Q} / 2+h_{\mathrm{r}} \times \alpha \sigma_{\mathrm{L}}
\end{gathered}
$$

Before the implementation of collaborative replenishment, the order quantity is determined by the retailer's minimum stock cost. Where $Q_{r}^{*}$ is used to represent the optimal order quantity before the collaborative replenishment. In order to obtain $Q_{r}^{*}$, we need to find the derivative of $T C_{r}$ to $Q$ and make $d T C_{r} / d Q=0$. According to the formula (6), there is certain relationship between $Q$ and $\alpha$. Get the derivative of $T C_{r}$ to $Q$ and get the result:

$$
\begin{gathered}
d T C_{r} / d Q=-c_{r} \mu / Q^{2}+h_{r} / 2+h_{r} \sigma_{L} d \alpha / d Q=0 \\
Q_{r}^{*}=\sqrt{2 c_{r} \mu /\left[h_{r}\left(1+2 \sigma_{L} \frac{d \alpha}{d Q}\right)\right]}
\end{gathered}
$$

(2) After the implementation of collaborative replenishment

The information sharing and data integration management between the retailer and the supplier have been achieved after the implementation of CPFR. After the collaborative planning and forecasting, the retailer's storage business and the ordering business are entrusted to the supplier at the replenishment stage. The supplier replaces the retailer to make replenishment decisions through real-time data exchange and inventory monitoring. Therefore, in the collaborative replenishment mode, the retailer's storage and ordering business costs are zero, which are all transferred to the supplier. The supplier's stock management cost is the total stock management fee of the supply chain. The supplier replaces the retailer and becomes the decision-maker of the replenishment strategy. The 
supplier makes the replenishment decision with the minimum cost of the supply chain stock management to determine the optimal order quantity.

The retailer's stock management cost is: $T C_{r}=0$

The supplier's stock management cost is:

$$
T C_{s}=c_{r} \times \frac{\mu}{Q}+h_{r} \times\left(Q / 2+\alpha \sigma_{L}\right)+c_{S} \mu / Q+h_{s} Q / 2
$$

The supply chain's total stock cost is:

$$
\begin{gathered}
T C=C_{r}+T C_{s}=c_{r} \mu / Q+h_{r}\left(Q / 2+\alpha \sigma_{L}\right)+c_{s} \mu / Q+h_{s} Q / 2 \\
=\left(c_{r}+c_{s}\right) \mu / Q+\left(h_{r}+h_{s}\right) Q / 2+h_{r} \times \alpha \sigma_{L}
\end{gathered}
$$

After the implementation of CPFR collaborative replenishment, because the supplier bears the supply chain stock cost, it will make the replenishment decision based on the minimum supply chain stock cost. Where $Q^{*}$ is used to represent the optimal order quantity after the collaborative replenishment. In order to obtain $Q^{*}$, we need to find the derivate of $T C$ to $Q$ and makedTC $/ d Q=0$.

$$
\begin{aligned}
d T C / d Q & =-\left(c_{r}+c_{s}\right) \mu / Q^{2}+\left(h_{r}+h_{s}\right) / 2+h_{r} \sigma_{L} d \alpha / d Q=0 \\
Q^{*} & =\sqrt{2\left(c_{r}+c_{s}\right) \mu /\left[h_{s}+h_{r}\left(1+2 \sigma_{L} d \alpha / d Q\right)\right]}
\end{aligned}
$$

Substitute $Q^{*}$ into the formula (12) and (13), and get the stock cost of the supplier as well as that of the supply chain after the implementation of collaborative replenishment.

In the analysis of the relationship between the model parameters, it has been concluded that there is a certain relationship between $Q$ and $\alpha$, but it is difficult to obtain $Q^{*}$ directly. It needs to be solved by the programming method.

In the above model, only two basic parameters change, namely the storage cost and the order cost. If the model is extended to the multi-level supply chain, only the two basic parameters need to be adjusted. The model structure is not affected. Therefore, the application of this model is quite extensive.

\section{Case Study}

This paper mainly uses the programming solution function in Excel to get the case solution.

(1) Set relevant parameters

Enter the variables, objective functions, constraints, and other known conditions into the table, as shown in Table 2.

Table 2. Related Parameter Value Setting

\begin{tabular}{|c|c|c|c|}
\hline Known Condition & Parameter & Value & Unit \\
\hline Retailer's Cost for One Order & $c_{r}$ & 100 & Yuan \\
\hline Supplier's Order Processing Cost Each Time & $c_{S}$ & 200 & Yuan \\
\hline Retailer's Unit Product Storage Cost & $h_{r}$ & 20 & Yuan \\
\hline Supplier's Unit Product Storage Cost & $h_{S}$ & 10 & Yuan \\
\hline The Expected Annual Demand & $\mu$ & 20000 & Piece \\
\hline Standard Deviation of Annual Demand & $\sigma$ & 600 & Piece \\
\hline Leading Time & $L$ & 7 & Day \\
\hline The Expected Demand at The Leading Time & $\mu_{L}$ & 384 & Piece \\
\hline The Standard Error of Demand at The Leading Time & $\sigma_{L}$ & 83 & Piece \\
\hline Product Satisfaction Rate & $\lambda$ & 0.95 & - \\
\hline
\end{tabular}

(2) Set the relationship formula

Before getting the solution, set the relationship of cells in the programming solution table according to the relationship of parameters in each formula of the model.

(3) Get the solution

Before the implementation of collaborative replenishment, the objective function is $T C_{r}$. After the implementation of collaborative replenishment, the objective function isTC. Before and after the implementation of collaborative replenishment, the variable parameters are $Q$ and $\alpha$, and the constraint is $\lambda=0.95$, namely $1-\frac{E(U)}{Q}=0.95$. Open the programming solution in Excel, and enter 
the above items, and get the minimum solution of the objective function. Because it is to solve the non-linear programming problem, choose "non-linear" from the dialog box of programming solution, and set it as "assumed non-negative".

After the solution, the best order quantity can be obtained, so do the corresponding $R, \alpha, T C_{r}, T C_{s}$, $T C$. The comparison of all calculation results before and after the replenishment is shown in Table 3.

Table 3. The Comparison of Results before and After the Collaborative Replenishment

\begin{tabular}{|c|c|c|c|c|}
\hline Programming Solution Item & Parameter & Before Collaborative Replenishment & $\begin{array}{c}\text { After Collaborative } \\
\text { Replenishment }\end{array}$ & \begin{tabular}{c} 
Unit \\
\hline Economical Order Batch
\end{tabular} \\
\hline Safety Coefficient & $\alpha$ & 512 & 647 & Piece \\
\hline Expected Order Quantity of Each Time & $E(U)$ & 0.20 & 32 & -01 \\
\hline Product Satisfaction Rate & $1-E(U) / Q$ & 26 & 0.95 & Piece \\
\hline Re-Order Point & $R$ & 400 & 384 & Piece \\
\hline The Retailer's Annual Stock Management Cost & $T C_{r}$ & 9357.00 & 0.00 & Yuan \\
\hline The Supplier's Annual Stock Management Cost & $T C_{s}$ & 10373.38 & 18989.19 & Yuan \\
\hline The Total Stock Management Cost of Supply Chain & $T C$ & 19730.39 & 18989.19 & Yuan \\
\hline
\end{tabular}

Through the comparison of results in Table 3, it can be seen that after the implementation of collaborative replenishment, under the same product satisfaction rate, the retailer and supplier can decrease the re-order point by increasing the order batch, thus reducing the frequency of ordering. Accordingly, it will achieve the goal of reducing the stock management cost of the whole supply chain, improving the collaboration of the supply chain, and enhancing both sides' competitiveness. The calculations above show that 9354.05 Yuan were saved for the retailer's stock cost after implementing the CPFR, and 736.42 Yuan were saved for the whole supply chain's stock cost. But it should be noted that the supplier's stock cost increased a lot. Therefore, after implementing the CPFR, the conflict of interests between node enterprises in the supply chain can be solved by transfer payment, discount, and increase of ordering rate.

\section{Sensitivity Analysis}

According to above calculation, the annul demand satisfies the normal distribution of $N(20000$, $600^{2}$ ). Here, assuming that the annual demand still satisfied the normal distribution, the standard deviation $\sigma$ is 11 groups of data with same interval from 500 to 1500 . According to the above relationship of parameters, calculate the corresponding $\sigma_{L}$, and other known conditions are unchanged. The constraint is still $\lambda=0.95$. Calculate the optimal replenishment quantity $Q$ and the stock cost of supply chain $T C$ before and after the collaborative replenishment.

It can be seen from Fig. 1, in case of a certain product satisfaction rate, with the increase in demand fluctuation, the optimal replenishment quantity before collaborative replenishment is gradually increased, while the optimal replenishment quantity changes slightly after collaborative replenishment. And they are both larger than the optimal replenishment quantity before implementing collaborative replenishment. It can be seen from Fig. 2 that after implementing the collaborative replenishment, at different levels of demand fluctuations, the total stock cost of supply chain is smaller than that before implementing the collaborative replenishment. And with the increase in demand fluctuations, the cost savings significantly increased.

Through the sensitivity analysis of demand fluctuation level, it can be explained that the implementation of collaborative replenishment can reduce the total stock cost of supply chain and the cost-saving effect tends to become more significant with the increase of demand fluctuation. Therefore, the node enterprises in the supply chain should strengthen the mutual collaboration, achieve information sharing, reduce the overall stock cost of supply chain, and at the same time improve the customer service level and increase profits. 


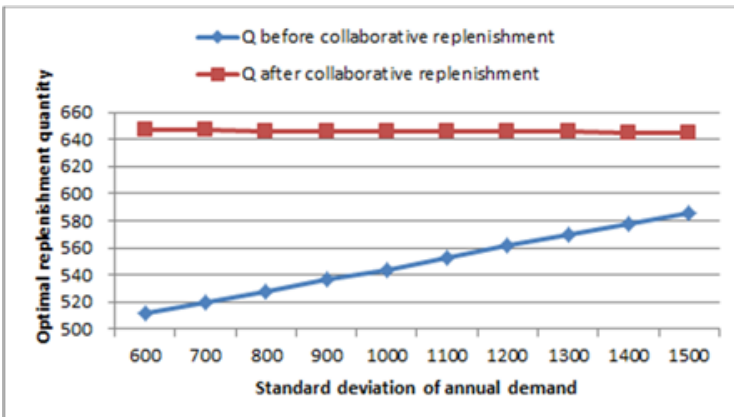

Figure 1. The Comparison of Optimal Replenishment Quantity before and after the Collaborative Replenishment at Different Demand Levels

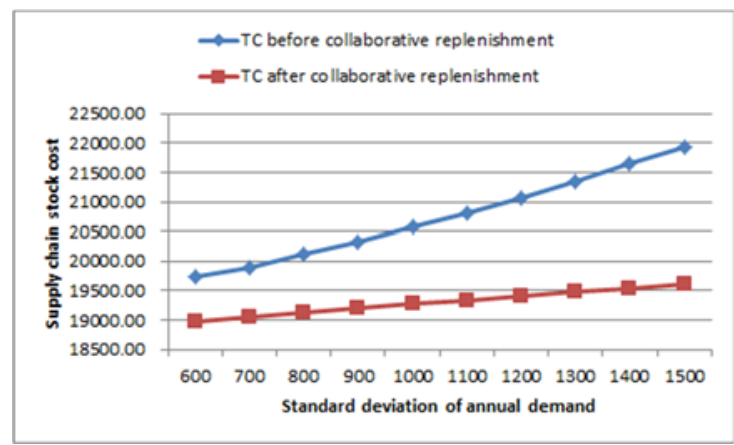

Figure 2. The Comparison of Supply Chain Stock Cost Before and After the Collaborative Replenishment at Different Demand Levels

\section{Conclusion}

CPFR has the advantage of achieving close cooperation between enterprises, improving the forecasting accuracy, improving customer service, exploring business opportunities, and taking full advantage of the efficiency of supply chain. It is a way to solve the problem of stock management.

The collaborative replenishment mode can help to save the stock management cost of the supply chain. And with the increase of demand and the rise of product satisfaction rate, more cost will be saved.

In this paper, the collaborative replenishment mode is analyzed. It proves that the collaborative replenishment mode can save stock management cost of the supply chain, enhance the information sharing and cooperation between node enterprises, strengthen the competitiveness and collaboration of the whole supply chain, and improve customer satisfaction. After implementing the CPFR in enterprise, it results in improved service level, increased service level, and reduced stock, and leads to the formation of a huge competitive advantage.

\section{References}

[1]. Danese, Collaboration Forms, Information and Communication Technologies, and Coordination Mechanisms in CPFR [J]. International Journal of Production Research, 2006, 44(6): 207-226.

[2]. Jerry Andrew, CPFR: Considering the Options, Advantages and Pitfalls [J]. Supply and Demand Executive, 2008, 4:8-12.

[3]. Susan Li, zhimin Huang, Allan Ashley, Manufacturer-retailer supply chain cooperation through franchising: a chance constrained game approach [J]. Info, 2005, 40(2):131-145.

[4]. Oliverira, A Exploring the experience of collaborative planning initiatives [J]. International Journal of Distribution \& Logistics Management, 2005, 17(4):266-289.

[5]. Steermainn, A Practical look at CPFR: The Sears-Michelin experience [J]. Supply Chain Management Review, 2005, 7(4):46. 
[6]. Lin, Rong-Ho, The study of CPFR implementation model in medical SCM of Taiwan [J]. Production Planning \& Control, 2014, 13(2):7-9.

[7]. Albright, CPFR s secret benefit [J]. Frontline Solutions, 2006, 3 (11):30-35.

[8]. Togar M. Simacupang, Ramaswami Sridharan, An integrative framework for supply chain collaboration [J], The International Journal of Logistics Management, 2007,16(2):257-274,

[9]. Sagar, CPFR at Whirlpool Corporation: Two heads and an exception engine [J]. The Journal of Business Forecasting Methods \& Systems, 2009, 22(4):3-8.

[10]. Bumette, Ron, CPFR: Fact, Fiction, or Fantasy [J]. The Journal of Business Forecasting, 2011, 29(4):32-35. 